\title{
Potential of microalga Isochrysis galbana: Bioactivity and bioaccessibility
}

\author{
Bonfanti C. ${ }^{\mathrm{a}, \mathrm{b}}$, Cardoso C. ${ }^{\mathrm{b}, \mathrm{c},}$, Afonso C. ${ }^{\mathrm{b}, \mathrm{c}, *}$, Matos J. ${ }^{\mathrm{b}, \mathrm{d}}$, Garcia T. ${ }^{\mathrm{a}, \mathrm{b}}$, Tanni S. ${ }^{\mathrm{a}}$, Bandarra N.M. ${ }^{\mathrm{b}, \mathrm{c}}$ \\ a Botucatu Medical School, UNESP - Universidade Estadual Paulista, Botucatu Campus, Botucatu, São Paulo, Brazil \\ b Division of Aquaculture and Upgrading (DivAV), Portuguese Institute for the Sea and Atmosphere (IPMA, IP), Rua Alfredo Magalhães Ramalho, 6, 1495-006 Lisbon, \\ Portugal \\ ${ }^{c}$ CIIMAR, Interdisciplinary Centre of Marine and Environmental Research, University of Porto, Rua dos Bragas 289, 4050-123 Porto, Portugal \\ ${ }^{\mathrm{d}}$ Faculdade de Ciências da Universidade de Lisboa, Campo Grande, 16, 1749-016 Lisbon, Portugal
}

\section{A R T I C L E I N F O}

\section{Keywords:}

Isochrysis galbana

Lipid composition

$\omega 3$ PUFA

Anti-inflammatory activity

Bioaccessibility

\begin{abstract}
A B S T R A C T
The lipid composition and anti-inflammatory activity of the microalga Isochrysis galbana were studied. Moreover, the influence of bioaccessibility on composition and bioactivity was evaluated through the application of an in vitro model of the human digestion. The fatty acid (FA) profile was characterized by abundance of polyunsaturated FA (PUFA) and, within PUFA, $\omega 3$ PUFA were the most abundant. High contents of myristic, oleic, linoleic, $\alpha$-linolenic, and stearidonic acids as well as docosahexaenoic acid (DHA) were determined. A low level of hydrolysis of triacylglycerols (TAGs) and polar lipids was observed during digestion. Total lipid bioaccessibility and specific FA bioaccessibility were low (between 7 and 15\%). The highest bioaccessibility percentages were determined for palmitic, oleic, and linoleic acids as well as total $\omega 6$ PUFA and the lowest bioaccessible percentages were calculated for myristic and stearidonic acids, DHA, and total $\omega 3$ PUFA. Chemical affinity phenomena could be an explanation for these results. Regarding anti-inflammatory activity, it was only detected in the lipid extract of $I$. galbana prior to digestion (79 $\pm 7 \%$ of cyclooxygenase-2, COX-2, inhibition). No activity was found in the bioaccessible fraction extract. Apparently, the COX-2 inhibitory compounds were not rendered bioaccessible.
\end{abstract}

\section{Introduction}

Microalgae are an important aquatic resource [1]. Moreover, microalgae are a promising and valuable natural source of bioactive compounds, such as $\omega 3$ polyunsaturated fatty acids ( $\omega 3$ PUFA). Due to its potential, microalgal biomass can be incorporated as a functional ingredient in order to enhance the nutritional value of foods and, thus, to positively affect human health.

In particular, the microalga Isochrysis galbana is potentially promising to the food industry due to its significantly high lipid content $(20-30 \% \mathrm{w} / \mathrm{dw})$, representing a rich source of $\omega 3$ PUFAs, namely eicosapentaenoic acid (EPA, 20:5 $\omega 3$ ) and docosahexaenoic acid (DHA, 22:6 $\omega 3$ ) [2]. Accordingly, it can be regarded as an additional source of essential oils to fisheries, thus covering the needs of increasing human population. It also supplies sterols, tocopherols, colouring pigments, and other bioactive substances [2,3].

EPA and DHA are associated with decreased morbidity and mortality from cardiovascular and other diseases as well as with foetal development [4]. Reviewed evidence also pointed to benefits for the development of the neural system in children [5] and prevention of mild cognitive decline in elderly [6]. Furthermore, EPA has been claimed to enhance anti-inflammatory properties of high-density lipoprotein, among other effects [7].

On the other hand, the reports of anti-inflammatory effects on rats due to I. galbana [8] may correspond to the action of bioactive substances in I. galbana, including EPA and other than EPA. These substances may be peptides, carotenoids, or sulphated polysaccharides [9]. In the case of the microalga Chlorella, evidence linking extracts of this organism to anti-inflammatory outcomes has been stronger $[1,10]$. More specifically, for the microalga Porphyridium cruentum, a sulphoglycolipidic fraction has been shown to display an anti-inflammatory effect [11]. A fatty acid (FA) analysis showed that this fraction contained large amounts of palmitic acid, 16:0 (26.1\%) and EPA (16.6\%) as well as noticeable amounts of the $16: 1 \omega 9(10.5 \%)$ [11]. These results show that microalgae have anti-inflammatory potential, which is stimulating the development of innovative nutraceuticals.

However, in future applications as nutraceuticals, it must be taken into account that the absorbable quantity of a compound in the gastrointestinal (GI) tract is not accurately predicted by its total content in

\footnotetext{
* Corresponding authors at: Division of Aquaculture and Upgrading (DivAV), Portuguese Institute for the Sea and Atmosphere (IPMA, IP), Rua Alfredo Magalhães Ramalho, 6, 1495-006 Lisbon, Portugal.

E-mail addresses: carlos.cardoso@ipma.pt (C. Cardoso), cafonso@ipma.pt (C. Afonso).
} 
the microalgae. Bioaccessibility corresponds to the share of the initial content that is rendered free from the microalgal structure into the GI tract [12]. Thus, determining bioaccessibility may contribute to the assessment of the effective nutraceutical potential of any given microalgal biomass. A bioaccessibility study requires the utilization of an adequate in vitro digestion model that reliably simulates human digestion. Different techniques have been developed [13] and optimized $[12,13]$, being the static model with digestive compartment distinction and complete digestive juices, including enzymes in all steps, one of the best models.

The objective of this study was to determine the lipid composition, anti-inflammatory activity, and bioaccessibility effects on these aspects for the microalgae I. galbana, paving the way for a deeper knowledge of the biochemical processes involved in any anti-inflammatory effects and a more realistic assessment of the nutraceutical potential.

\section{Material and methods}

\subsection{Sample collection and preparation}

Samples of Isochrysis galbana were supplied by Necton (Necton, Companhia Portuguesa de Culturas Marinhas, SA, Olhão, Portugal). This material was already freeze-dried and was analysed without further processing.

\subsection{Proximate composition}

The moisture and ash contents were determined according to Association of Official Analytical Chemists (AOAC) methods [14]. The protein level was quantified according to the Dumas method [15]. Crude lipid content was determined following the Bligh and Dyer extraction method [16]. Carbohydrate content was determined by difference.

\subsection{Fatty acid profile}

Fatty acid methyl esters (FAME's) were prepared by acid-catalysed transesterification using the methodology described by Bandarra et al. [17]. Samples were injected into a Varian Star 3800 CP gas chromatograph (Walnut Creek, CA, USA), equipped with an auto sampler with a flame ionisation detector at $250^{\circ} \mathrm{C}$. FAME's were identified by comparing their retention time with those of Sigma-Aldrich standards (PUFA-3, Menhaden oil, and PUFA-1, Marine source from Supelco Analytical). The limit of detection (LOD) is $1 \mathrm{mg} / 100 \mathrm{~g}$. Results were calculated in $\mathrm{mg} / 100 \mathrm{~g}$ of edible part using the peak area ratio (\% of total fatty acids) and the lipid conversion factors, which were, in turn, calculated according to Weihrauch et al. [18].

\subsection{Lipid class determination}

The relative weight of each lipid class was determined by analytical thin-layer chromatography (TLC) using a previously described method [19]. An eluent mixture of hexane:diethyl ether:acetic acid (50:50:2 by volume) and a plate coated with $0.25 \mathrm{~mm}$ silica gel $\mathrm{G}$ were used. Lipid class identification was done by comparison with standards (Sigma Chemical Co., St. Louis, MO, USA). Specifically, glyceryltrioleate (triacylglycerol, TAG), glyceryl 1,3-dipalmitate (diacylglycerol, DAG), DL- $\alpha$-monoolein (monoacylglycerol, MAG), oleic acid (free fatty acid, FFA), and L- $\alpha$-phosphatidylcholine (phospholipid, PL, polar lipid) were used. The relative percentage of each lipid class was determined using a GS-800 densitometer and version 4.5.2 of Quantity One 1-D Analysis software from Bio-Rad (Hercules, CA, USA).

\subsection{Anti-inflammatory activity}

To extract lipids from freeze-dried I. galbana microalgae and respective bioaccessible fraction, the Bligh and Dyer method [16] and the methodology described in Section 2.7. were used, respectively. Extracted lipids were directly dissolved in $100 \%$ dimethyl sulfoxide (DMSO) (see below Section 2.5.2).

\subsubsection{Aqueous extract preparation for in vitro anti-inflammatory activity}

An aqueous extract of the freeze-dried I. galbana microalgae was prepared with the purpose of attaining a fraction with anti-inflammatory properties to be tested in vitro.

Accordingly, approximately $200 \mathrm{mg}$ of freeze-dried I. galbana microalgae was weighed and homogenized with $2 \mathrm{ml}$ of Milli-Q water using a model Polytron PT 6100 homogenizer (Kinematica, Luzern, Switzerland) at a velocity of 30,000 rpm during $1 \mathrm{~min}$. Afterwards, the mixture was subjected to a thermal treatment (at $80^{\circ} \mathrm{C}$ for $1 \mathrm{~h}$ ). Both the microalgae and bioaccessible extraction mixtures were centrifuged $\left(3000 \times g\right.$ at $4{ }^{\circ} \mathrm{C}$ during $10 \mathrm{~min}$ ) and the respective supernatant was evaporated using vacuum rotary evaporator with the water bath temperature at $65^{\circ} \mathrm{C}$ and inert gas (nitrogen) stream.

\subsubsection{Cyclooxygenase (COX-2) inhibition assay}

The prepared extracts were dissolved in $100 \%$ DMSO to prepare a stock with a concentration of $10 \mathrm{mg} / \mathrm{ml}$. The extract was tested at $1 \mathrm{mg} / \mathrm{ml}$ and $100 \mu \mathrm{g} / \mathrm{ml}$ using a commercial cyclooxygenase (COX-2) inhibitory screening assay kit, Cayman test kit-560,131 (Cayman Chemical Company, Ann Arbor, MI, USA). The COX-2 inhibitor screening assay directly measures the amount of Prostaglandin F2 $\alpha$ generated from arachidonic acid (AA, 20:4 $\omega 6$ ) in the cyclooxygenase reaction. A volume of $10 \mu \mathrm{l}$ each of test extract or DMSO was used. The reaction was initiated by addition of $10 \mu 110 \mathrm{mM}$ AA and each reaction tube was incubated at $37^{\circ} \mathrm{C}$ for $2 \mathrm{~min}$. The reaction was terminated by addition of $50 \mu \mathrm{l} 1 \mathrm{~N} \mathrm{HCl}$ and saturated stannous chloride. Assays were performed using 100 units of human recombinant COX-2. An aliquot was removed and the prostanoid produced was quantified spectrophotometrically $(412 \mathrm{~nm}$ ) via enzyme immunoassay (ELISA) after $18 \mathrm{~h}$ incubation, washing, addition of Ellman's reagent, and further $90 \mathrm{~min}$ incubation. Interference by solutions and digestive enzymes used in the bioaccessibility method was taken into account by subtracting COX-2 inhibition of the bioaccessibility blank from the COX-2 inhibition measured with the bioaccessible fraction samples.

\subsection{In vitro digestion model}

An in vitro digestion model was chosen for the determination of bioaccessibility in freeze-dried I. galbana microalgae. The model was comprised of three sections, which enable the simulation of digestion in three different parts of the GI tract: mouth, stomach, and small intestine. The solutions and enzymes used in this model followed Afonso et al. [12]. Briefly, approximately $1.5 \mathrm{~g}$ freeze-dried I. galbana was weighed taking into account the assumptions defined by Versantvoort et al. [20]. The sample was mixed with $4 \mathrm{ml}$ of artificial saliva at a pH $6.8 \pm 0.2$ for $5 \mathrm{~min}$, then $8 \mathrm{ml}$ of artificial gastric juice $\left(\mathrm{pH} 1.3 \pm 0.02\right.$ at $\left.37 \pm 2{ }^{\circ} \mathrm{C}\right)$ was added, and $\mathrm{pH}$ was lowered to $2.0 \pm 0.1$. The mixing lasted $2 \mathrm{~h}$ in a "head-over-heels" movement (37 rpm at $37 \pm 2{ }^{\circ} \mathrm{C}$ ). Finally, $8 \mathrm{ml}$ of artificial duodenal juice $\left(\mathrm{pH} 8.1 \pm 0.2\right.$ at $\left.37 \pm 2{ }^{\circ} \mathrm{C}\right), 4 \mathrm{ml}$ of bile $(\mathrm{pH} 8.2 \pm 0.2$ at $37 \pm 2{ }^{\circ} \mathrm{C}$ ), and $1.33 \mathrm{ml}$ of $\mathrm{HCO}_{3}{ }^{-}$solution $(1 \mathrm{M})$ was added. The $\mathrm{pH}$ of the mixture was set at $6.5 \pm 0.5$ and agitation for $2 \mathrm{~h}$ was identical to gastric conditions. The mixture generated in the in vitro model was subjected to centrifugation at $2750 \times g$ for $5 \mathrm{~min}$, thus yielding a nondigested portion and the bioaccessible fraction. While chemicals were supplied by Merck (Darmstadt, Germany), enzymes were attained from Sigma (St. Louis, MO, USA).

\subsubsection{Calculation of bioaccessibility}

The percentage (\%) of each I. galbana microalgae constituent (C) in the bioaccessible fraction was estimated as follows: 
$\%$ C bioaccessible $=[C]_{\text {bioaccessible }} \times 100 /[\mathrm{S}]$

Being:

[C] = Concentration of constituent.

$[\mathrm{S}]=[\mathrm{C}]$ in the initial sample (before digestion).

\subsection{Lipid extraction in the bioaccessible and undigested fractions}

For bioaccessible samples, the Bligh and Dyer method [16] was slightly modified since the lipid fraction was rendered available by the digestion procedure. Four milliliters chloroform was added to the bioaccessible fraction followed by $1 \mathrm{~min}$ homogenisation in a vortex and a centrifugation at $2000 \times g$ for $5 \mathrm{~min}$. Then, the upper phase was removed. In the next step, $4 \mathrm{ml}$ chloroform and $2 \mathrm{ml}$ of water were added followed by $1 \mathrm{~min}$ homogenisation in a vortex and a centrifugation at $2000 \times \mathrm{g}$ for $5 \mathrm{~min}$ at a temperature of $4{ }^{\circ} \mathrm{C}$. After the removal of the upper phase, the previous operation was repeated. The organic phase was then filtered through a filter containing anhydrous sodium sulphate and then evaporated in a rotary evaporator. The lipid samples were weighed, solubilised in chloroform and stored at $-20{ }^{\circ} \mathrm{C}$ until further analysis. For the undigested fraction, the procedure was just as described by Bligh and Dyer [16].

\subsection{Statistical analysis}

To test the normality and the homogeneity of variance of data, the Kolmogorov-Smirnov's test and Levene's F-test, respectively, were used. Data, which corroborate these assumptions, were analysed by one-way Anova distribution using the Tukey HSD to determine the difference in the content of constituents between the initial and the bioaccessible fraction of samples or between fatty acids. When normality and/or homogeneity of variance were not verified (initial 18:0 content and $\omega 3$ / $\omega 6$ vs bioaccessible 18:0 content and $\omega 3 / \omega 6$ ), data were tested nonparametrically with Kruskal-Wallis test and Mann-Whitney's $U$ test. For all statistical tests the significance level $(\alpha)$ was 0.05 . All data analysis was performed using STATISTICA 6 (Stat-sof, Inc. USA, 2003).

\section{Results and discussion}

\subsection{Microalgal characterization}

\subsubsection{Proximate composition}

The proximate composition of studied I. galbana species is displayed in Table 1. Moisture content was low $(7.7 \pm 0.0 \%, \mathrm{w} / \mathrm{w})$ owing to the fact that studied microalgae was freeze-dried. The dry matter of this microorganism was mainly composed of protein and lipids, $42.3 \pm 1.2 \%, \mathrm{w} / \mathrm{w}$, and $25.3 \pm 0.2 \%, \mathrm{w} / \mathrm{w}$, respectively. Nonetheless, ash and carbohydrate fractions were also a significant share of the biomass.

The observed proximate composition was similar to that reported by other authors for I. galbana [21,22]. Indeed, the biomass of this microorganism is very rich in lipids, even in comparison with other lipidrich microalgae, such as Diacronema vlkianum [22].

\subsubsection{Lipid composition}

The distribution of lipid classes in the lipid fraction of I. galbana microalgae is presented in Table 2 and the fatty acid composition of $I$. galbana microalgae is shown in Table 1.

There were two main lipid classes in the studied I. galbana biomass: triacylglycerols (TAGs) and free fatty acids (FFAs). Polar lipids represented a relatively small share of $10.2 \pm 0.7 \%$ of total lipids.

For lipid-rich biomass (lipid content exceeding 5\%,w/w), TAGs are usually the most abundant class in fish and other organisms [23], but these empirical relations are not necessarily applicable in microalgae [24]. These authors found a high share (exceeding 80\%) of polar lipids in Isochrysis sp. However, there are other authors who have reported a
Table 1

Proximate composition (\% wet weight) and fatty acid profile (in \% of total fatty acids and in $\mathrm{mg} / 100 \mathrm{~g}$ wet weight) of the studied microalgae I. galbana before and after digestion (bioaccessible fraction).

\begin{tabular}{llll}
\hline Microalgae before digestion & & & Microalgae after digestion \\
\cline { 1 - 2 } Proximate composition & (\% wet weight) & & (Bioaccessible fraction) \\
\hline Moisture & $7.7 \pm 0.0$ & \\
Lipid & $25.3 \pm 0.2$ & \\
Protein & $42.3 \pm 1.2$ & \\
Carbohydrate & $9.9 \pm 1.2$ & \\
Ash & $14.8 \pm 0.1$ & \\
& &
\end{tabular}

\begin{tabular}{|c|c|c|c|c|}
\hline \multicolumn{3}{|c|}{ Microalgae before digestion } & \multicolumn{2}{|c|}{ Microalgae after digestion } \\
\hline Fatty acid & $\begin{array}{l}\text { (\% total fatty } \\
\text { acids) }\end{array}$ & $\begin{array}{l}\text { (mg/100 g wet } \\
\text { weight) }\end{array}$ & $\begin{array}{l}\text { (\% total fatty } \\
\text { acids) }\end{array}$ & $\begin{array}{l}\text { (mg/100 g } \\
\text { wet weight) }\end{array}$ \\
\hline $14: 0$ & $14.1 \pm 0.1^{\mathrm{A}}$ & $3311 \pm 30$ & $11.2 \pm 0.7^{\mathrm{B}}$ & $16.9 \pm 1.0$ \\
\hline $16: 0$ & $10.6 \pm 0.0^{\mathrm{A}}$ & $2489 \pm 6$ & $12.9 \pm 0.9^{\mathrm{A}}$ & $19.4 \pm 1.2$ \\
\hline 18:0 & $0.5 \pm 0.0^{\mathrm{A}}$ & $127 \pm 1$ & $3.0 \pm 0.6^{\mathrm{B}}$ & $4.6 \pm 0.9$ \\
\hline$\Sigma \mathrm{SFA}^{\mathrm{a}}$ & $27.8 \pm 0.2^{\mathrm{A}}$ & $6513 \pm 40$ & $29.4 \pm 1.2^{\mathrm{A}}$ & $44.2 \pm 1.4$ \\
\hline $16: 1 \omega 7$ & $5.0 \pm 0.0^{\mathrm{A}}$ & $1174 \pm 7$ & $4.9 \pm 0.1^{\mathrm{A}}$ & $7.4 \pm 0.1$ \\
\hline $18: 1 \omega 9$ & $14.7 \pm 0.1^{\mathrm{A}}$ & $3452 \pm 15$ & $17.4 \pm 0.4^{\mathrm{B}}$ & $26.2 \pm 0.9$ \\
\hline $20: 1 \omega 11$ & $0.9 \pm 0.0^{\mathrm{A}}$ & $214 \pm 8$ & $1.0 \pm 0.0^{\mathrm{A}}$ & $1.5 \pm 0.1$ \\
\hline$\Sigma$ MUFA $^{\mathrm{b}}$ & $23.0 \pm 0.2^{\mathrm{A}}$ & $5389 \pm 37$ & $25.1 \pm 0.3^{\mathrm{B}}$ & $37.8 \pm 0.9$ \\
\hline $18: 2 \omega 6$ & $9.6 \pm 0.0^{\mathrm{A}}$ & $2245 \pm 5$ & $11.4 \pm 0.2^{\mathrm{B}}$ & $17.2 \pm 0.4$ \\
\hline $20: 4 \omega 6$ & $0.3 \pm 0.0^{\mathrm{A}}$ & $69 \pm 3$ & $1.0 \pm 0.0^{\mathrm{B}}$ & $1.5 \pm 0.1$ \\
\hline $18: 3 \omega 3$ & $10.9 \pm 0.0^{\mathrm{A}}$ & $2557 \pm 5$ & $10.5 \pm 0.1^{\mathrm{B}}$ & $15.8 \pm 0.3$ \\
\hline $18: 4 \omega 3$ & $11.3 \pm 0.0^{\mathrm{A}}$ & $2644 \pm 9$ & $10.3 \pm 0.3^{\mathrm{B}}$ & $15.5 \pm 0.6$ \\
\hline $20: 4 \omega 3$ & $0.2 \pm 0.0^{\mathrm{A}}$ & $37 \pm 0$ & $0.2 \pm 0.0^{\mathrm{A}}$ & $0.2 \pm 0.0$ \\
\hline $20: 5 \omega 3$ & $1.2 \pm 0.0^{\mathrm{A}}$ & $276 \pm 1$ & $1.1 \pm 0.1^{\mathrm{A}}$ & $1.7 \pm 0.1$ \\
\hline $22: 5 \omega 3$ & $0.2 \pm 0.0^{\mathrm{A}}$ & $42 \pm 3$ & $0.3 \pm 0.1^{\mathrm{A}}$ & $0.4 \pm 0.1$ \\
\hline $22: 6 \omega 3$ & $9.2 \pm 0.2^{\mathrm{A}}$ & $2146 \pm 42$ & $8.0 \pm 0.3^{\mathrm{B}}$ & $12.1 \pm 0.7$ \\
\hline$\Sigma$ PUFA $^{\mathrm{c}}$ & $46.2 \pm 0.4^{\mathrm{A}}$ & $10,829 \pm 85$ & $45.5 \pm 0.9^{\mathrm{A}}$ & $68.7 \pm 2.1$ \\
\hline$\Sigma \omega 3$ & $33.6 \pm 0.3^{\mathrm{A}}$ & $7867 \pm 61$ & $30.7 \pm 0.8^{\mathrm{B}}$ & $46.2 \pm 1.6$ \\
\hline$\Sigma \omega 6$ & $12.1 \pm 0.1^{\mathrm{A}}$ & $2829 \pm 22$ & $14.3 \pm 0.3^{\mathrm{B}}$ & $21.6 \pm 0.6$ \\
\hline$\Sigma \omega 3 / \Sigma \omega 6$ & $2.8 \pm 0.0^{\mathrm{A}}$ & $2.8 \pm 0.0$ & $2.1 \pm 0.1^{\mathrm{B}}$ & $2.1 \pm 0.1$ \\
\hline
\end{tabular}

Values are presented as average \pm standard deviation. Different uppercase letters within a row correspond to statistical differences $(p<0.05)$ between microalgae before and after digestion (bioaccessible fraction).

${ }^{a}$ SFA, saturated fatty acid.

${ }^{\mathrm{b}}$ MUFA, monounsaturated fatty acid.

${ }^{\mathrm{c}}$ PUFA, polyunsaturated fatty acid.

high percentage of TAGs [25]. The large share of FFAs may be due to different causes: effects of culture medium composition on microalgal metabolism [21]; lipid breakdown in stressed or dying cells [24]; and hydrolytic degradation of the biomass, which may have occurred during frozen storage ( 9 months at $-20{ }^{\circ} \mathrm{C}$ ) [26]. Studies on I. galbana [21] have reported a wide variability in the proportion of polar lipids and neutral lipids as a result of changes in the cultivation conditions and time of harvest, that is, a dependence on the different phases of the growth cycle. A high proportion of TAGs has been correlated with the absence of cellular division at the onset of stationary phase in aged cultures of microalgae [27].

Regarding the FA profile, two main aspects may be underlined: on the one hand, PUFAs were the main FA group $(46.2 \pm 0.4 \%$ of total FAs), followed by saturated FAs (SFA, $27.8 \pm 0.2 \%$ ), and monounsaturated FAs (MUFA, $23.0 \pm 0.2 \%$ ); on the other hand, within the PUFA group, $\omega 3$ PUFAs were clearly more abundant than $\omega 6$ PUFAs, thereby yielding an $\omega 3 / \omega 6$ ratio of nearly 3 .

Other aspects are also worth mentioning at a more detailed level: myristic acid (14:0) was the most abundant SFA (14.1 $\pm 0.1 \%)$; oleic acid (18:1 $\omega 9)$ represented more than half total MUFA (14.7 $\pm 0.1 \%)$; almost all $\omega 6$ PUFAs were linoleic acid (18:2 $\omega 6)$; and $\omega 3$ PUFAs were mainly composed of similar proportions, in the 9-11\% range, of $\alpha$-linolenic acid (18:3 $\omega 3$ ), stearidonic acid (18:4 $\omega 3$ ), and DHA. EPA content was very low, barely exceeding $1 \%$ of total FAs. 
Table 2

Lipid class distribution (\% of total lipid) before and after digestion of the studied microalgae $I$. galbana and in other studies.

\begin{tabular}{|c|c|c|c|c|c|c|}
\hline \multirow[t]{2}{*}{ Microalgae } & \multirow[t]{2}{*}{ Initial vs Bioaccessible } & \multicolumn{4}{|l|}{ Lipid Classes } & \multirow[t]{2}{*}{ Source } \\
\hline & & TAG* & $\mathrm{FFA}^{* *}$ & Polar Lipids & Other & \\
\hline \multirow[t]{2}{*}{ I. galbana } & Initial & $34.5 \pm 0.3^{\mathrm{a}}$ & $39.8 \pm 1.0^{\mathrm{a}}$ & $10.2 \pm 0.7^{\mathrm{a}}$ & $15.5 \pm 1.4^{\mathrm{a}}$ & Current study \\
\hline & Bioaccessible & $26.3 \pm 4.5^{\mathrm{a}}$ & $54.0 \pm 5.7^{b}$ & $7.5 \pm 0.4^{\mathrm{b}}$ & $12.2 \pm 1.6^{\mathrm{a}}$ & Current study \\
\hline I. galbana & Initial & $26-61$ & - & $39-74$ & - & {$[21]$} \\
\hline Isochrysis sp. & Initial & 2.8 & $<0.2$ & 83.0 & 14.1 & {$[24]$} \\
\hline I. galbana & Initial & $>50$ & - & $<40$ & - & [25] \\
\hline N. oculata & Bioaccessible & - & $<55$ & - & - & [33] \\
\hline
\end{tabular}

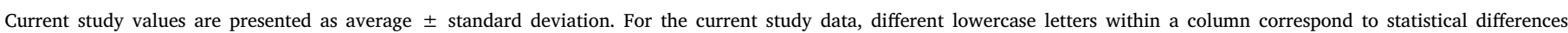
$(p<0.05)$.

$*$ TAG, triacylglycerol.

*** FFA, free fatty acid.

The FA profile of $I$. galbana microalgae can vary widely as a function of cultivation conditions and growth phase at harvest time [28]. For instance, an inverse relation it has been observed between EPA and DHA levels and growth temperature in Isochrysis, as well as in other microalgae [29]. In particular, EPA contents may experience wide variations between batches with different cultivation conditions [28]. Some traits experience smaller changes, such as the abundance of myristic acid $[21,22,28]$. High levels of stearidonic acid, such as those observed in current study, have also been measured by other authors [21]. Some aspects, such as the high oleic and linoleic acids levels and low EPA level, are not readily found in the literature [21,22,28].

Moreover, taking into account human EPA + DHA requirements and considering that I. galbana may be used in food applications, the amount of freeze-dried microalga needed to meet the recommended daily intake of EPA + DHA (500 mg/day) according to the American Heart Association may be calculated [30]. On the basis of the absolute FA composition in Table 1 , an amount of $20.6 \mathrm{~g}$ of the studied freezedried I. galbana can be estimated, which is a low amount and may enable the incorporation of this microalga in foods. In addition, it should be stressed that given the fact that high EPA + DHA levels before digestion (especially DHA in I. galbana) do not ensure a high intestinal absorption of these lipids, a study on microalgal lipid digestion is warranted (see below).

\subsection{Microalgal lipid digestion}

\subsubsection{Lipid hydrolysis}

The level of lipid hydrolysis achieved in the bioaccessible fraction can be assessed by the distribution of lipid classes, as displayed in Table 2.

There was a significant level of TAGs remaining after digestion (26.3 $\pm 4.5 \%$ of total lipids). FFAs increased with respect to the initial sample (prior to digestion), but by only $15 \%$, reaching $54.0 \pm 5.7 \%$. Polar lipids were slightly reduced from $10.2 \pm 0.7 \%$ to $7.5 \pm 0.4 \%$ of total lipid.

Accordingly, these results indicated a low hydrolysis of TAGs and polar lipids. This contrasts with other studies claiming a high level of lipid hydrolysis with no TAGs detected in the bioaccessible fraction $[12,23,31,32]$. However, all these studies were on fish and not based on microalgae. Unfortunately, there are very few studies on microalgal lipid bioaccessibility $[13,33]$. The latter authors also reported low lipolysis levels in another species of microalga, Nannochloropsis oculata, at least, in the absence of prior treatment of the microalgal biomass. Regarding polar lipids, though it has been claimed that these amphiphilic lipids modify the surface structure of oil droplets in such a way that access to lipids by pancreatic lipases is enhanced [34], no extensive microalgal polar lipid lipolysis was detected. Nevertheless, it should be noted that for some polar lipids, such as phosphatidylcholine, total lipolysis is not necessary for bioaccessibility [35].

\subsubsection{Bioaccessible fatty acids}

The FA profile of the bioaccessible fraction of the studied microalgae I. galbana is presented in Table 1 and, on the basis of the comparison between the FA profile before and after digestion (bioaccessible fraction), the bioaccessibility percentages were calculated for the total lipids and each FA, being shown in Fig. 1.

There were some differences between the FA profile before digestion (Table 1) and in the bioaccessible fraction. However, the overall proportion of PUFA and SFA was constant. Within the SFA group, the palmitic acid content surpassed that of myristic acid, which was reduced in comparison to the FA content in the microalga prior to digestion, thereby suggesting differences in the degree of bioaccessibility of this SFA. Regarding MUFA, the relative abundance of oleic acid in the bioaccessible fraction was higher than in the initial sample,
A

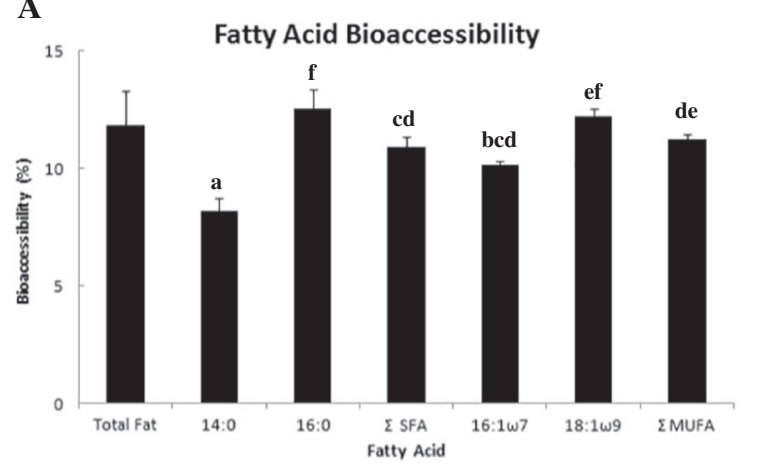

B

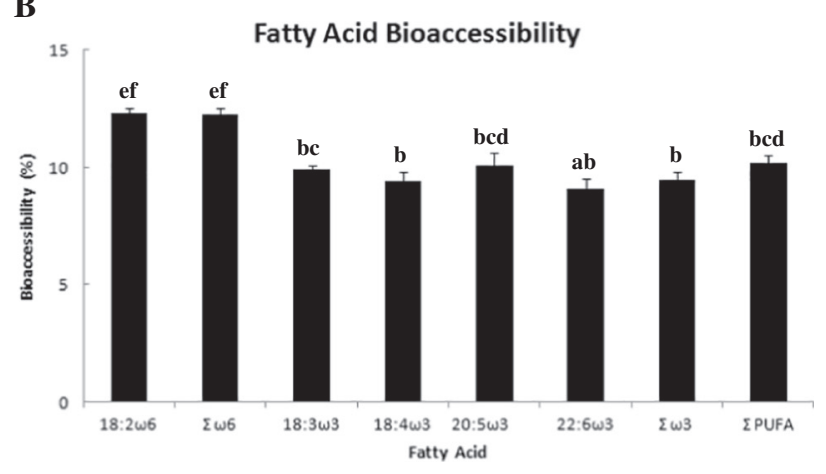

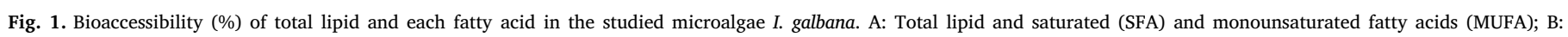
Polyunsaturated fatty acids (PUFA).

Different lowercase letters correspond to statistical differences between fatty acids $(p<0.05)$. 
exceeding $17 \%$ of the total FAs. A similar increase was also determined for the two main $\omega 6$ PUFAs, linoleic acid and AA, entailing a corresponding increase in the total $\omega 6$ PUFA level. On the other hand, the stearidonic acid and DHA contents were slightly reduced after digestion. The other $\omega 3$ PUFAs, as a percentage of total FAs, were left unchanged. Hence, the total $\omega 3$ PUFA content in the bioaccessible fraction was lower than in I. galbana biomass before digestion, $30.7 \pm 0.8 \%$ vs $33.6 \pm 0.3 \%$ of total FAs. The opposite variations of total $\omega 6$ PUFA and total $\omega 3$ PUFA entailed a decrease of the $\omega 3 / \omega 6$ ratio to only $2.1 \pm 0.1$ in the bioaccessible fraction of I. galbana.

These variations had obvious implications for the bioaccessibility percentages. Both total lipid bioaccessibility and each particular FA bioaccessibility were low and within a narrow range of approximately 7-15\%. The highest bioaccessibility percentages were determined for palmitic, oleic, and linoleic acids, as well as total $\omega 6$ PUFA, thus, agreeing with the relative enrichment of these FAs in the FA profile of the bioaccessible fraction. On the other end of the spectrum of variation, the lowest bioaccessible percentages were calculated for myristic and stearidonic acids, DHA, and total $\omega 3$ PUFA. This also matched the observed variations in the FA profile of the bioaccessible fraction with respect to the initial (before digestion) FA profile. The remaining FAs exhibited intermediate bioaccessibility percentages.

First, it should be highlighted that the results show the importance of studying human digestion of microalgal lipids, given the observed significant variations. For instance, $\omega 6$ PUFAs were more available for intestinal absorption than $\omega 3$ PUFAs. Nevertheless, the deviations with respect to the initial FA profile of I. galbana were not large. For fish (sole), a study on lipid bioaccessibility showed large variations to the $\omega 3$ PUFA content prior to digestion [31]. Just as in the current study, $\omega 3$ PUFA level was lower in the bioaccessible fraction of sole. Since FA in vitro bioaccessibility in the whole microalgal biomass was not previously researched and investigation into microalgal foods is still a nascent area [36], there are only studies on other matrices, such as fish, as a reference.

There are also some studies on microalgal FA apparent digestibility - relating FA contents in the diet and feces, thereby enabling an estimate of the share of the initial FA content in the microalga that is assimilated by the organism - through in vivo animal experiments with salmon [37]. This study used untreated microalgal biomass (Schizochytrium sp.) and found evidence supporting high microalgal digestibility for lipids in general and $\omega 3$ PUFA in particular. This seems to oppose the low FA bioaccessibility (including $\omega 3$ PUFA) in I. galbana, but it should be noticed that salmon feed is extruded - extrusion pressure and temperature may favour cell disruption - and there are differences between microalgae species [38]. This deserves further investigation, since the possibility of using unprocessed microalgal biomass directly in animal and human nutrition would be advantageous over extracting microalgal oil (economic costs in extraction, loss of nutrients, and higher risk of oxidation of $\omega 3$ PUFA). Specifically, aquaculture is in urgent need of adequate volumes of new $\omega 3$ PUFA sources [37], more sustainable in the long term than fish oil and less expensive than microalgal oil.

The percentages of lipid and FA bioaccessibility in I. galbana were very low and unprecedented, if compared with other bioaccessibility studies on fish lipids and using a similar in vitro model $[12,23,31,32]$. In these studies, lipid bioaccessibility was always above $50 \%$ - salmon and gilthead seabream - $[23,32]$ or $29 \%$ - meagre and sole $[12,31]$. Therefore, there seems to be a higher difficulty in digesting microalgal biomass. Indeed, a study on $N$. oculata [33] showed that neither proteins nor lipids of intact microalgal cells were accessible to the mammalian digestive enzymes used in the applied digestion model. There was only some improvement in lipid digestion after microalgal treatment by $\mathrm{pH}$-shift processing (from $\mathrm{pH} 7.0$ to $\mathrm{pH} 10.0$ ). A possible explanation was the microalgal cell wall composition of Nannochloropsis, consisting predominantly of cellulose, surrounded by an outer layer of algaenan, which may block enzymes from acting on the cell [39]. It is also known that algal cell walls change during growth and if microalgae are subjected to nitrogen stress [40]. However, Isochrysis genus lacks a cell wall, only possessing a plasma membrane covering [41]. For this reason, utilization of Isochrysis in nutrition has been advised as easily assimilated biomass. Accordingly, the explanation for the low lipid bioaccessibility in I. galbana must lie elsewhere. It is possible that chemical interaction phenomena play a role in these bioaccessibility results. In this case, microalgal lipids would have displayed higher affinity for the non-bioaccessible fraction components, which could comprise carbohydrates and other components (Table 1). Furthermore, the low degree of lipolysis and high level of remaining TAGs (Table 2) suggest inhibition of the digestive model lipases by some component(s) in the microalgal biomass. These hypotheses and this lipid bioaccessibility study on microalgae need to be supported by further experimental work.

Taking into account the very low bioaccessibility, the differences between bioaccessibility percentages of each FA were not very significant. Nonetheless, some differences, such as a higher bioaccessibility percentage of oleic acid and a lower bioaccessibility percentage of DHA and $\omega 3$ PUFA, have been found in fish matrices [23,32]. Chemical structure aspects may underlie these differences. It has been reported that higher level of unsaturation leads to lower bioaccessibility percentage [23] and DHA and other $\omega 3$ PUFA are highly unsaturated.

Another consequence of the low lipid bioaccessibility is a lower nutritional value regarding $\omega 3$ PUFA content. Effectively, for achieving the recommended daily intake of $\mathrm{EPA}+\mathrm{DHA}$ according to the American Heart Association, $500 \mathrm{mg} /$ day [30], a much higher quantity of freeze-dried I. galbana, approximately ten times more, must be ingested than otherwise calculated (20.6 g). Of course, a serving portion of $200 \mathrm{~g}$ of freeze-dried microalgae or the incorporation of this quantity in a functional food is unviable. Therefore, it is of paramount importance to find options that enhance lipid bioaccessibility in I. galbana. Such options would enable to take advantage of anti-inflammatory substances, EPA [7] and other, present in the I. galbana biomass (see below).

\subsection{Microalgal anti-inflammatory activity}

Anti-inflammatory activity may result from the inhibition of enzymes - such as cyclooxygenase (COX-2) - involved in the conversion of fatty acids into prostaglandins and leukotrienes, which display an inflammatory action $[42,43]$. Accordingly, the anti-inflammatory activity values of the I. galbana biomass measured as a percentage of inhibition of the enzyme COX-2 were determined and are presented in Table 3. Two different extracts of the initial (prior to digestion) freezedried biomass were tested, an aqueous and an oily extract. Furthermore, the anti-inflammatory activity of the bioaccessible fraction (oily extract) was also determined. Extract concentration was $5 \mathrm{mg} / \mathrm{ml}$ in DMSO.

Anti-inflammatory activity was only detected in the oily extract of the initial I. galbana biomass, reaching a value of $79 \pm 7 \%$ of COX-2 inhibition. For the bioaccessible fraction extract - after eliminating the bioaccessibility blank background interference - and the other (aqueous) initial extract, no activity was detected.

Table 3

Anti-inflammatory activity (\% inhibition of cyclooxygenase-2, COX-2) in the studied microalgae $I$. galbana before (initial, in aqueous and lipid extract) and after digestion (bioaccessible, in lipid extract).

\begin{tabular}{lll}
\hline & Extract & Anti-inflammatory activity (\% inhibition of COX-2) \\
\hline Initial & Aqueous & $\mathrm{nd}^{\mathrm{a}}$ \\
& Lipid & $79 \pm 7^{\mathrm{b}}$ \\
Bioaccessible & Lipid & $\mathrm{nd}^{\mathrm{a}}$
\end{tabular}

nd - Not detected. Values are presented as average \pm standard deviation. Different lowercase letters within a column correspond to statistical differences $(p<0.05)$. 
There are some studies on the anti-inflammatory activity of microalgae, but methodologies are different, ranging from in vitro assays to in vivo models [44-46]. This makes comparison among studies difficult. Nevertheless, several studies point to the existence of anti-inflammatory activity in microalgae $[44,46]$. Whereas some authors found significant activity in aqueous extracts, for instance, in $\mathrm{Ar}$ throspira platensis [44], other authors have reported anti-inflammatory properties of lipid components and precisely in I. galbana [46].

In general, different components may be involved, such as phenolic compounds, carotenoids, phytosterols, alkaloids, polysaccharides or proteins, such as phycocyanin [44]. However, in the current study, lipids and lipophilic substances seem to be at the root of the anti-inflammatory activity, since activity was only detected in the lipid extract. Besides EPA, which may have anti-inflammatory properties [7], other lipid fraction substances in I. galbana biomass may contribute to the anti-inflammatory properties. Indeed, it should also be remarked that even though EPA and $\omega 3$ PUFA have anti-inflammatory effects [47], their action usually requires in vivo systems, which was not the case in the COX-2 inhibition assay used in current study. A recent study [46] identified glycolipids, such as digalactosyldiacylglycerols, as inhibitors of the production of pro-inflammatory cytokine in human THP1 macrophages. The COX-2 inhibition reported in the current study seems to point to another anti-inflammatory mechanism, thus justifying further study on the nature of the COX-2 inhibitor(s) in the oily fraction of I. galbana.

Such inhibitor(s) did not seem to be bioaccessible. If a low bioaccessibility of the anti-inflammatory compounds in I. galbana is confirmed, preparation of extracts for nutraceutical applications or microalgal processing through decoction to produce tisane or other alternatives [33] may be advantageous, thereby rendering these compounds more bioaccessible.

\section{Conclusions}

The biomass of the studied I. galbana was rich in protein and lipids. The FA profile was characterized by abundance of PUFA and, within PUFA, $\omega 3$ PUFA were the most abundant. High contents of myristic, oleic, linoleic, $\alpha$-linolenic, and stearidonic acids as well as DHA were determined. Lipid, particularly FA, bioaccessibility was studied through the application of an in vitro model. A low hydrolysis of TAGs and polar lipids during digestion was observed. Moreover, both total lipid bioaccessibility and each particular FA bioaccessibility were low (between 7 and 15\%). The highest bioaccessibility percentages were determined for palmitic, oleic, and linoleic acids as well as total $\omega 6$ PUFA and the lowest bioaccessible percentages were calculated for myristic and stearidonic acids, DHA, and total $\omega 3$ PUFA. Chemical affinity phenomena may be at the root of these results. Concerning anti-inflammatory activity, it was only detected in the oily extract of I. galbana biomass prior to digestion ( $79 \pm 7 \%$ of COX-2 inhibition). No activity was detected in the bioaccessible fraction extract. Future work should focus on preparing extracts for nutraceutical applications or microalgal processing through decoction (tisane) for achieving a higher bioaccessibility of lipid components and anti-inflammatory bioactives.

\section{Acknowledgments}

This work received financial support from CAPES for Carolina Bonfanti (88881.134961/2016-01). This work was also supported by the following Post-Doctoral Grants: Ref.: SFRH/BPD/102689/2014 ("Fundação para a Ciência e a Tecnologia", FCT) for the author Carlos Cardoso; Ref.: SFRH/BPD/64951/2009 (FCT) and DIVERSIAQUA (MAR2020, Ref.: 16-02-01-FEAM-66) for the author Cláudia Afonso. A doctoral grant awarded by FCT (SFRH/BD/129795/2017) supported the work performed by Joana Matos. The experimental work was funded by the project Algared + (0055_ALGARED_PLUS_5_E) "Rede
Transfronteiriça para o Desenvolvimento de Produtos Inovadores com Microalgas".

\section{Conflict of interest}

No conflict of interest involving any of the authors.

\section{Authors contributions}

The conception and design of the study was carried out by S Tanni and NM Bandarra, the acquisition of data and its analysis was done by $\mathrm{C}$ Bonfanti and $\mathrm{T}$ Garcia, and the interpretation of data was done by $\mathrm{C}$ Afonso and J Matos. The drafting the article and its critical revision for important intellectual content was performed by C Cardoso. Its final version was approved by NM Bandarra.

\section{Statement of informed consent, human/animal rights}

No conflicts, informed consent, human or animal rights applicable.

\section{References}

[1] J. Matos, C. Cardoso, N.M. Bandarra, C. Afonso, Microalgae as a healthy ingredient for functional food: a review, Food Funct. 8 (2017) 2672-2685.

[2] A.P. Batista, L. Gouveia, N.M. Bandarra, J.M. Franco, A. Raymundo, Comparison of microalgal biomass profiles as novel functional ingredient for food products, Algal Res. 2 (2013) 164-173.

[3] L. Gouveia, A.E. Marques, J.M. Sousa, P. Moura, N.M. Bandarra, Microalgae source of natural bioactive molecules as functional ingredients, Food Sci. Technol. Bull.: Funct. Foods 7 (2) (2010) 21-37.

[4] A.P. Simopoulos, Omega-3 fatty acids and cardiovascular disease: the epidemiological evidence, Environ. Health Prev. Med. 6 (2002) 203-209.

[5] C. Cardoso, C. Afonso, N.M. Bandarra, Dietary DHA, bioaccessibility, and neurobehavioural development in children, Crit. Rev. Food Sci. Nutr. (2017).

[6] C. Cardoso, C. Afonso, N.M. Bandarra, Dietary DHA and health: Cognitive function aging, Nutr. Res. Rev. 29 (2016) 281-294.

[7] N. Tanaka, T. Ishida, M. Nagao, T. Mori, T. Monguchi, M. Sasaki, K. Mori, K. Kondo, H. Nakajima, T. Honjo, Y. Irino, R. Toh, M. Shinohara, K. Hirata, Administration of high dose eicosapentaenoic acid enhances anti-inflammatory properties of highdensity lipoprotein in Japanese patients with dyslipidemia, Atherosclerosis 237 (2) (2014) 577-583.

[8] K. Nuño, A. Villarruel-López, A.M. Puebla-Pérez, E. Romero-Velarde, A.G. PueblaMora, F. Ascencio, Effects of the marine microalgae Isochrysis galbana and Nannochloropsis oculata in diabetic rats, J. Funct. Foods 5 (1) (2013) 106-115.

[9] E. Talero, S. García-Mauriño, J. Ávila-Román, A. Rodríguez-Luna, A. Alcaide, V. Motilva, Bioactive compounds isolated from microalgae in chronic inflammation and cancer, Mar. Drugs 13 (2015) 6152-6209.

[10] S. Guzmán, A. Gato, M. Lamela, M. Freire-Garabal, J.M. Calleja, Anti-inflammatory and immunomodulatory activities of polysaccharide from Chlorella stigmatophora and Phaeodactylum tricornutum, Phytother. Res. 17 (2003) 665-670.

[11] J.P. Bergé, E. Debiton, J. Dumay, P. Durand, C. Barthomeuf, In vitro anti-inflammatory and anti-proliferative activity of sulfolipids from the red alga Porphyridium cruentum, J. Agric. Food Chem. 50 (2002) 6227-6232.

[12] C. Afonso, S. Costa, C. Cardoso, N.M. Bandarra, I. Batista, I. Coelho, I. Castanheira, M.L. Nunes, Evaluation of the risk/benefit associated to the consumption of raw and cooked farmed meagre based on the bioaccessibility of selenium, eicosapentaenoic acid and docosahexaenoic acid, total mercury, and methylmercury determined by an in vitro digestion model, Food Chem. 170 (2015) 249-256.

[13] C. Cardoso, C. Afonso, H. Lourenço, S. Costa, M.L. Nunes, Bioaccessibility assessment methodologies and their consequences for the risk-benefit evaluation of food, Trends Food Sci. Technol. 41 (2015) 5-23.

[14] AOAC, Official Methods of Analysis of the AOAC International, 17th ed, Association of Analytical Communities, Gaithersburg, 2000.

[15] T. Saint-Denis, J. Goupy, Optimization of a nitrogen analyser based on the dumas method, Anal. Chim. Acta 515 (1) (2004) 191-198.

[16] E. Bligh, W. Dyer, A rapid method of total lipid extraction and purification, Can. J. Biochem. Physiol. 37 (1959) 911-917.

[17] N.M. Bandarra, I. Batista, M.L. Nunes, J.M.A. Empis, W.W. Christie, Seasonal changes in lipid composition of sardine Sardina pilchardus, J. Food Sci. 62 (1) (1997) 40-43.

[18] J.L. Weihrauch, L.P. Posati, B.A. Anderson, J. Exler, Lipid conversion factors for calculating fatty acids contents in foods, JAOCS 54 (1977) 36-40.

[19] N.M. Bandarra, I. Batista, M.L. Nunes, J.M. Empis, Seasonal variation in the chemical composition of horse-mackerel (Trachurus trachurus), Eur. Food Res. Technol. 212 (2001) 535-539.

[20] C.H.M. Versantvoort, A.G. Oomen, E. Van de Kamp, C.J. Rompelberg, A.J. Sips, Applicability of an in vitro digestion model in assessing the bioaccessibility of mycotoxins from food, Food Chem. Toxicol. 43 (2005) 31-40. 
[21] J.P. Fidalgo, A. Cid, E. Torres, A. Sukenik, C. Herrero, Effects of nitrogen source and growth phase on proximate biochemical composition, lipid classes and fatty acid profile of the marine microalga Isochrysis galbana, Aquaculture 166 (1998) 105-116.

[22] M. Fradique, A.P. Batista, M.C. Nunes, L. Gouveia, N.M. Bandarra, A. Raymundo, Isochrysis galbana and Diacronema vlkianum biomass incorporation in pasta products as PUFA's source, LWT 50 (2013) 312-319.

[23] S. Costa, C. Afonso, C. Cardoso, I. Batista, N. Chaveiro, M.L. Nunes, N.M. Bandarra, Fatty acids, mercury, and methylmercury bioaccessibility in salmon (Salmo salar) using an in vitro model: effect of culinary treatment, Food Chem. 185 (2015) 268-276.

[24] J.K. Volkman, S.W. Jeffrey, P.D. Nichols, G.I. Rogers, C.D. Garland, Fatty acid and lipid composition of 10 species of microalgae used in mariculture, J. Exp. Mar. Biol. Ecol. 128 (1989) 219-240.

[25] G. Breuer, P.P. Lamers, D.E. Martens, R.B. Draaisma, R.H. Wijffels, The impact of nitrogen starvation on the dynamics of triacylglycerol accumulation in nine microalgae strains, Bioresour. Technol. 124 (2012) 217-226.

[26] J.A. Nelson, Postharvest degradation of microalgae: Effect of temperature and water activity. All graduate theses and dissertations. 4458. https://digitalcommons.usu. edu/etd/4458.

[27] P.A. Hodgson, R.J. Henderson, J.R. Sargent, J.W. Leftley, Patterns of variation in the lipid class and fatty acid composition of Nannochloropsis oculata (Eustigmatophyceae) during batch culture: I. The growth cycle, J. Appl. Phycol. 3 (1991) 169-181.

[28] Y. Durmaz, M. Donato, M. Monteiro, L. Gouveia, M.L. Nunes, T.G. Pereira, S. Gokpınar, N.M. Bandarra, Effect of temperature on growth and biochemical composition (sterols, $\alpha$-tocopherol, carotenoids, fatty acid profiles) of the microalga, Isochrysis galbana, Isr. J. Aquacult. Bamidgeh 60 (3) (2008) 190-197.

[29] A. Tasselli, M. Doimi, The polyunsaturated fatty acid of thermically treated phytoplankton, Il Pesce 2 (1990) 33-37.

[30] P. Kris-Etherton, W. Harris, L. Appel, American Heart Association Nutrition Committee Fish consumption, fish oil, omega-3 fatty acids, and cardiovascular disease, Circulation 106 (2002) 2747-2757.

[31] C. Afonso, C. Cardoso, M. Freire, I.E. Silva, F. Linares, J.L.R. Villanueva, L.M.P. Valente, N.M. Bandarra, The impact of alternative dietary lipids on the in vitro bioaccessibility of sole fillets for human consumption, Aquaculture 474 (2017) 66-74.

[32] S. Costa, C. Afonso, C. Cardoso, R. Oliveira, F. Alves, M.L. Nunes, N.M. Bandarra, Towards a deeper understanding of fatty acid bioaccessibility and its dependence on culinary treatment and lipid class: a case study of gilthead seabream (Sparus aurata), Br. J. Nutr. 116 (2016) 1816-1823.

[33] L.R. Cavonius, E. Albers, I. Undeland, In vitro bioaccessibility of proteins and lipids of pH-shift processed Nannochloropsis oculata microalga, Food Funct. 7 (2016)
2016-2024.

[34] S. Mun, E.A. Decker, D.J. McClements, Influence of emulsifier type on in vitro digestibility of lipid droplets by pancreatic lipase, Food Res. Int. 40 (2007) 770-781.

[35] J.S. Cohn, A. Kamili, E. Wat, R.W.S. Chung, S. Tandy, Dietary phospholipids and intestinal cholesterol absorption, Nutrients 2 (2) (2010) 116-127.

[36] M.L. Wells, P. Potin, J.S. Craigie, J.A. Raven, S.S. Merchant, K.E. Helliwell, A.G. Smith, M.E. Camire, S.H. Brawley, Algae as nutritional and functional food sources: revisiting our understanding, J. Appl. Phycol. 29 (2) (2017) 949-982.

[37] K. Kousoulaki, T. Mørkøre, I. Nengas, R.K. Berge, J. Sweetman, Microalgae and organic minerals enhance lipid retention efficiency and fillet quality in Atlantic salmon (Salmo salar L.), Aquaculture 451 (2016) 47-57.

[38] D. Gangl, J.A. Zedler, P.D. Rajakumar, E.M. Martinez, A. Riseley, A. Włodarczyk, S. Purton, Y. Sakuragi, C.J. Howe, P.E. Jensen, C. Robinson, Biotechnological exploitation of microalgae, J. Exp. Bot. 66 (22) (2015) 6975-6990.

[39] M.J. Scholz, T.L. Weiss, R.E. Jinkerson, J. Jing, R. Roth, U. Goodenough, M.C. Posewitz, H.G. Gerken, Ultrastructure and composition of the Nannochloropsis gaditana cell wall, Eukaryot. Cell 13 (11) (2014) 1450-1464.

[40] H.G. Gerken, B. Donohoe, E.P. Knoshaug, Enzymatic cell wall degradation of Chlorella vulgaris and other microalgae for biofuels production, Planta 237 (1) (2013) 239-253.

[41] C.J. Zhu, Y.K. Lee, T.M. Chao, Effects of temperature and growth phase on lipid and biochemical composition of Isochrysis galbana TK1, J. Appl. Phycol. 9 (1997) 451-457.

[42] J. Oestvang, B. Johansen, Phospholipase A2: a key regulator of inflammatory signaling and a connector to fibrosis development in atherosclerosis, Biochim. Biophys. Acta Mol. Cell Biol. Lipids 1761 (11) (2006) 1309-1316.

[43] A. George, S. Chinnappan, M. Chintamaneni, V.C. Kotak, Y. Choudhary, T. Kueper, A.K. Radhakrishnan, Anti-inflammatory effects of Polygonum minus (Huds) extract (Lineminus ${ }^{\mathrm{TM}}$ ) in in-vitro enzyme assays and carrageenan induced paw edema, BMC Complement. Altern. Med. 14 (2014) 355.

[44] G.S. Jensen, V.L. Attridge, J.L. Beaman, J. Guthrie, A. Ehmann, K.F. Benson, Antioxidant and anti-inflammatory properties of an aqueous cyanophyta extract derived from Arthrospira Platensis: contribution to bioactivities by the non-phycocyanin aqueous fraction, J. Med. Food 18 (5) (2015) 535-541.

[45] G.L. Renju, K.G. Muraleedhara, K.C.H. Saritha, Anti-inflammatory activity of lycopene isolated from Chlorella marina on type II collagen induced arthritis in Sprague Dawley rats, Immunopharmacol. Immunotoxicol. 35 (2) (2013) 282-291.

[46] C. Reyes, M.J. Ortega, A. Rodríguez-Luna, E. Talero, V. Motilva, E. Zubia, Molecular characterization and anti-inflammatory activity of galactosylglycerides and galactosylceramides from the microalga Isochrysis galbana, J. Agric. Food Chem. 64 (46) (2016) 8783-8794.

[47] P.C. Calder, Omega-3 fatty acids and inflammatory processes, Nutrients 2 (3) (2010) 355-374. 